SILVA, M. B. R.; BATISTA, R. C.; LIMA, V. L. A.; BARBOSA, E. M.; BARBOSA, M. F. N. Crescimento de plantas jovens da espécie florestal favela (Cnidosculus phyllacanthus Pax \& K. Hoffm.) em diferentes níveis de salinidade da água.

Revista de Biologia e Ciências da Terra, v.5, n.2 sp., jul./dez., 2005.

SILVA, M. B. R.; VIÉGAS, R. A.; DANTAS NETO, J.; FARIAS, S. A. R. Estresse salino em plantas da espécie florestal sabiá. Caminhos de Geografia, v. 10, n. 30, p. 120-127, jun., 2009.

SINGH, G.; SINGH, T.; BHOJVAID, P. P. Amelioration of sodic soils by tree for wheat and oat production. Land Degradation \& Development, v. 9 , n. 5 , p. $453-462$, sept./oct., 1998.

SOUZA, R. S. Potencial de espécies florestais nativas na fitoextração de sais. 2013. 63f. Dissertação (Mestrado em Ciências Florestais e Ambientais) Universidade Federal de Mato Grosso, Cuiabá, 2013.

SU, N.; BETHUNE, M.; MANN, L.; HEUPERMAN, A. Simulating water and salt movement in tile drained fields irrigated with saline water under a Serial Biological Concentration Management Scenario. Agricultural Water Management, v. 78, n. 3, p. 165-180, dec., 2005.

TAIZ, L.; ZEIGER, E. Fisiologia vegetal. 3.ed. Porto Alegre: ARTMED, 2004. 719p

TOBE, K.; LI, X.; OMASA, K. Seed germination and radicle growth of a halophyte, Kalidium capsicum (Chenopodiaceae). Annals of Botany, v. 85, n. 3, p. 391-396, 2000.

VAN HOORN, J. W.; KATERJI, N.; HAMDY, A.; MASTRORILLI, M. Effect of salinity on yield and nitrogen uptake of four legumes and on biological nitrogen contribution from the soil. Agricultural Water Management, v. 51, n. 2, p. 87-98, oct., 2001.

VIEIRA, D. B. As técnicas de irrigação. São Paulo: Globo, 1989. 283p.

\section{IDENTIFICAÇÃO DE FUNGOS TOXIGÊNICOS E SUAS \\ RESPECTIVAS TOXINAS EM UVAS PASSAS ESCURAS COMERCIALIZADAS EM VOTUPORANGA-SP}

\author{
Ana Fernandes Santos ${ }^{1}$ \\ Yasmim Martins Silva \\ Cátia Rezende \\ Christiane de Oliveira Jordão ${ }^{1}$
}

\section{RESUMO}

As micotoxinas são metabólitos secundários produzidos por alguns fungos filamentosos que possuem propriedades tóxicas. A principal via de exposição a essa toxina é normalmente através da ingestão de alimentos contaminados, mesmo que sejam em pequenas quantidades, mas de forma contínua podem levar ao seu acúmulo no organismo podendo causar uma intoxicação e afetando muitos órgãos e sistemas, principalmente o fígado, rins e sistema nervoso. As micotoxinas mais frequentemente detectadas são produzidas por fungos dos gêneros Aspergillus, Penicillium e Fusarium. As uvas passas são produtos obtidos a partir da perda parcial da água da fruta madura por secagem, apresentando alto teor de açúcar e baixa atividade de água, fato que favorece o crescimento de fungos, principalmente A. niger e A. carbonarius que produzem esporos resistentes ao processamento das mesmas. Este estudo teve por objetivo realizar o isolamento e identificação de fungos produtores de micotoxinas e suas respectivas toxinas em uvas passas escuras a granel e embaladas no próprio estabelecimento comercial, utilizando os métodos de microcultivo e cromatografia em camada delgada comparativa. Foram detectadas a presença de A. niger, Penicillium spp e A. flavus, os primeiros, produtores de ocratoxinas e o último de aflatoxinas. Portanto, a pesquisa demonstrou a contaminação por fungos toxigênicos nas amostras coletadas em $82 \%$ dos estabelecimentos avaliados.

Palavras-chave: Uvas Passas; Intoxicação; Micotoxinas. 


\section{IDENTIFICATION OF TOXIGENIC FUNGI AND ITS RESPECTIVE TOXINS IN DARK RAISINS COMMERCIALIZED IN VOTUPORANGA- SP}

\section{ABSTRACT}

Mycotoxins are secondary metabolites produced by some filamentous fungi that have toxic properties. The main way of exposure to that toxin is usually through the ingestion of contaminated food even if it is in small quantity but continuously it can induce to its accumulation in the body and cause an intoxication, affecting many organs and systems, especially the liver, kidneys and the nervous system. The most detected mycotoxins are produced by fungi from the genus Apergillus, Penicillium and Fusarium. The raisins are products obtained from the partial loss of water from the ripe fruit by drying, presenting high content of sugar and low activity of water, fact that favors the growth of fungi, principally A. niger and A. carbonarius which produce resistant spores to processing. This study aimed to achieve the isolation and identification of fungi which produce the mycotoxin and its respective toxins in dark raisins in bulk and packaged in the commercial establishment itself using microculture methods and thin layer chromatography. The presence of A. niger and Penicillium spp were detected, the first ones being producers of ocratoxina and A. flavus, the producer of aflatoxina. Therefore the research showed the contamination by toxigenic fungi in the collected samples in $82 \%$ of the evaluated establishments.

Keywords: Dried Fruit; Intoxication; Mycotoxins.

\section{INTRODUÇÃO}

Os metabólitos secundários produzidos por alguns fungos filamentosos que possuem propriedades tóxicas são chamados de micotoxinas. A principal via de exposição a essa toxina é normalmente através da ingestão de alimentos contaminados apesar de existirem casos de inalação e absorção dérmica (MASLINKIEWCZ \& FREITAS, 2012)

A contaminação do alimento varia de acordo com as condições do ambiente (luz, oxigênio, temperatura, $\mathrm{pH}$ e umidade do ar), do manuseio, armazenamento e transporte (SANTOS; LOPES; KOSSEKI, 2001; BATATINHA et al., 2003).

A ingestão destas substâncias tóxicas mesmo em pequenas quantidades de forma contínua pode levar ao seu acúmulo no organismo causando uma intoxicação (KOVACS, 2004) podendo afetar muitos órgãos e sistemas, principalmente o fígado, muitos órgãos e sistemas, principalmente o fígado, rins, sistema nervos
(FREIRE et al., 2007)

As micotoxinas mais frequentemente detectadas são produzidas por fungos dos gêneros Aspergillus, Penicillium e Fusarium (WELKE et al., 2009).

De acordo com as informações de Council for Agricultural Science and Technology (2003) as micotoxinas de maior incidência na contaminação humana são aflatoxinas, fumonisinas, tricotecenos, ocratoxina A e zearalenona. Outras micotoxinas são consideradas menos importantes, devido à incidência limitada em humanos.

As aflatoxinas e ocratoxinas são produzidas pelo gênero Aspergillus, facilmente encontrados em alimentos como grãos, legumes, café, frutas secas, leite, aveia e uvas (PERRONE et al., 2007; PEREYRA et al., 2010).

Apenas as aflatoxinas possuem níveis máximos em alimentos estabelecidos pela legislação. $\mathrm{O}$ Ministério da Saúde estabelece o limite de $30 \mu \mathrm{g}$ $\mathrm{kg}$ de AFB1 e AFG1. Apesar desta não prever níveis máximos de ocratoxinas em alimentos, e importante que programas de monitoramento acompanhem estudos de exposição humana para avaliar a necessidade de estabelecer níveis destas micotoxinas (CALDAS; SILVA; OLIVEIRA. 2002).

Em uvas os fungos encontrados são Aspergillus niger e Aspergillus carbonarius podendo contaminar tais frutas durante os processos de maturação, colheita, armazenamento e transporte (FAVILLA et al., 2008).

A presença de A. niger e A. carbonarius em uvas e produtos derivados, como suco de uva, uvas passas e vinhos, contribui para a contaminação por ocratoxina, pois esta pode resistir aos processos industriais sofridos pelo produto (OLIVEIRA; OLIVEIRA; MENEGHELLO, 2013).

As aflatoxinas são produzidas por cincos espécies de Aspergillus que possuem grande importância em alimentos, afinal constituem- se risco para a saúde dos consumidores. Sendo estes $A$. parasiticus, A. fumigatus, A. ochraceus, A. nomius (Food Ingredients Brasil, 2009).

KWIATKOWSKI \& ALVES (2007) referem à existência de 18 compostos designados como aflatoxinas, porém, os principais tipos de interesse médico são identificados como B1 (AFB1), B2, G1 e G2. Entre estas, as de maior toxicidade são as AFB1 e AFG1. O A. flavus produz apenas o grupo $\mathrm{B}$, enquanto as espécies A, parasiticus e A. nomius produzem os grupos B2 e G2. "Quando observadas em luz ultravioleta (UV), essas micotoxinas fluorescem nas seguintes colorações: B1 e B2- azul, G1- verde e G2-verde azulada As ocratoxinas se dividem em A, B e C; Ocratoxina A C apresentam fluorescência verde e B azul" (Food Ingredients Brasil, 2009).

Alimentos susceptíveis a contaminação incluem amendoim (cru, torrado, creme, em doce e confeitado), milho (pipoca, canjica e grãos) trigo, arroz, castanha de caju, amendoas, frutas secas, temperos, sementes de algodão, mandioca, óleos vegetais, cacau, entre outros que normalmente são utilizados na composição de alimentos (KWIATKOWSKI \& ALVES, 2007).

Frutas secas são produtos obtidos à partir da perda parcial da água da fruta madura por processo 
de secagem. Por apresentarem alto teor de açúcar e baixa atividade de água, acabam favorecendo o crescimento de fungos. Além disso, A. nige e A. carbonarius por produzirem esporos, são resistentes a raios solares, persistindo nas frutas durante a secagem ao sol durante a secagem ao (MENEGHELO; JORGE;

OLIVEIRA, 2012 apud IAMANAKA, 2004).

Tendo em vista os argumentos acima relatados, pode-se inferir que uvas passas à granel e embaladas no próprio estabelecimento estão mais expostas ao meio ambiente externo, tornando-as mais susceptíveis à contaminação por fungos. Assim sendo, o presente trabalho teve por objetivo isolar e identificar fungos produtores e suas respectivas toxinas em amostras de uvas passas escuras comercializadas em supermercados da cidade de Votuporanga- SP.

\section{METODOLOGIA}

Foram coletadas 22 amostras de uvas passas escuras em 11 pontos de venda do comércio de Votuporanga, São Paulo, no período de Maio à Junho de 2014. Foram analisadas 6 amostras de uvas passas escuras embaladas no próprio estabelecimento comercial e 5 vendidas à granel no mês de Maio. As análises foram repetidas no mês de Junho, com 7 amostras embaladas e 4 à granel.

Uma vez coletadas, estas foram transferidas para sacos plásticos estéreis a fim de se evitar qualquer tipo qualquer tipo de contaminação e encaminhada ao laboratório de análises clínicas e de química do Centro Universitário de Votuporanga- UNIFEV, em temperatura ambiente. O procedimento utilizado para o isolamento e a identificação de fungos produtores de toxinas foi baseado na metodologia descrita por FRANCO \& LANDGRAF (2000) descrís po Hiluic̃ã cultivo contendo Ágar Sabouraud e Cloranfenicol. O microcultivo foi realizado conforme SIDRIM \& MOREIRA (1999). A identificação de toxinas fúngicas foi baseada em MOREAU \& SIQUEIRA (2008), utilizando se técnica de cromatografia em camada delgada comparativa com o uso de revelador físico e químico.

\section{Isolamento, identificação}

e microcultivo dos fungos

Cada amostra coletada compunha-se de $200 \mathrm{~g}$ de uvas passas à granel e embaladas, analisadas em até 36 horas após sua obtenção (FRANCO \& LANDGRAF 2001). No momento da análise, a superfície externa do saco plástico foi desinfetada com álcool 70\% e gaze, em fluxo laminar. Posterion $25 \mathrm{~g}$ de cada amostra foram transferidos para balão volumétrico com 225 $\mathrm{mL}$ de solução salina peptonada $1 \%$ (diluição $10^{-1}$ ). A partir desta suspensão foram realizadas duas diluições seriadas $\left(10^{-2}, 10^{-3}\right)$ com o mesmo diluente. Das diluições foram retirados $10 \mu \mathrm{L}$ e espalhados em ágar Sabouraud dextrosado adicionado de cloranfenicol, por técnica de cultivo em superfície. Após o crescimento das colônias foram feitos os microcultivos, em ágar Batata dextrose, incubados à temperatura ambiente por ate 15 dias. As lâminas para análises microscópica das estruturas de reprodução foram coradas com lactofenol azul de algodão segundo ZAITZ, RUIZ e SOUZA (2004).

\section{Identificação de micotoxinas por romatografia em camada delgada comparativa (CCDC)}

A extração e purificação das micotoxinas foram realizadas no laboratório de química do Centro Universitário de Votuporanga- UNIFEV. Por meio de método de quarteamento de $200 \mathrm{~g}$ de uvas passas escuras amostraram se $50 \mathrm{~g}$, que transformadas em uma pasta foram extraídas com metanol e solução de KCL 4\%. Seguiu se o processo de clarificação da mesma com cloreto de cobre a $10 \%$ e $5 \mathrm{~g}$ de celite. A fase hidroalcoólica resultante extraiu se com 10 $\mathrm{ml}$ de clorofórmio em triplicata.

Para a identificação das micotoxinas por cromatografia em camada delgada o resíduo obtido foi dissolvido novamente em clorofórmio e aplicado em dois pontos da placa de sílica em gel 60 GF 254 $\mathrm{nm}\left(\right.$ Merck $\left.{ }^{\circledR}\right)$. A eluição das placas foi feitas com os seguintes sistemas solventes tolueno, acetato de etila e ácido fórmico (6:3:1), tolueno, acetato de etila e ácido ácetico (8:1:1). A revelação das placas foi feita utilizando se luz ultravioleta $(\lambda 365 \mathrm{~nm})$, observando-se fluorescência azul ou verde. Após a revelação fisica, as placas foram bor A reação com ácido serve para confirmar a presença de aflatoxinas pelo aparecimento de manchas com fluorescência amarela sob luz ultravioleta.

\section{RESULTADOS E DISCUSSÃO}

As uvas passas encontradas nas prateleiras de vários estabelecimentos comerciais podem esconder riscos à saúde do consumidor. Entre os 11 estabelecimentos avaliados identificou-se a presença de fungos produtores de micotoxinas em nove deles. Foram analisadas 11 amostras no mês de maio e junho para os mesmos estabelecimentos comerciais, totalizando 22 amostras. Destas $13(59 \%)$ apresentaram resultado positivo para micotoxinas e $9(41 \%)$ apresentaram resultado negativo, sendo que 6 apresentaram resultado positivo no mês de maio e 7 amostras positivas no mês de junho, todas as amostras se encontravam dentro do prazo de validade. Os resultados obtidos das análises microbiológicas do mês de maio e junho estão descritos na seguinte tabela (Tabela 1).

Tabela 1- Fungos filamentosos e prováveis toxinas identificadas em amostras de uvas passas escuras à granel e embaladas no próprio estabelecimento em 11 pontos comerciais de Votuporanga, S.P, durante os meses de maio e junho de 2014

\begin{tabular}{c|c|c|c|c}
\hline & \multicolumn{2}{|c|}{ MAIO } & \multicolumn{2}{c}{ JUNHO } \\
\hline ESTABELECIMENTO & FUNGO & $\begin{array}{c}\text { TOXINA } \\
\text { SUGESTIVA }\end{array}$ & FUNGO & $\begin{array}{c}\text { TOXINA } \\
\text { SUGESTIVA }\end{array}$ \\
\hline $\mathrm{A}$ & Negativo & Negativo & A. niger & Ocratoxina \\
\hline $\mathrm{B}$ & Mucor spp & - & A. niger & Ocratoxina \\
\hline $\mathrm{C}$ & A. niger & Ocratoxina & A. niger & Ocratoxina \\
\hline $\mathrm{D}$ & Negativo & Negativo & Negativo & Negativo \\
\hline $\mathrm{E}$ & Negativo & Negativo & Negativo & Negativo \\
\hline $\mathrm{F}$ & Negativo & Negativo & A. niger & Ocratoxina \\
\hline $\mathrm{G}$ & A. flavis & Aflatoxina & A.niger & Ocratoxina \\
\hline $\mathrm{H}$ & Penicillium spp & Ocratoxina & Negativo & Negativo \\
\hline $\mathrm{I}$ & A. niger & Ocratoxina & A. niger & Ocratoxina \\
\hline $\mathrm{J}$ & A. niger & Ocratoxina & A. niger & Ocratoxina \\
\hline $\mathrm{K}$ & A. niger & Ocratoxina & Negativo & Negativo \\
\hline
\end{tabular}


Tabela 2 Relação dos fungos produtores de micotoxinas identificados por microcultivo e fluorescências emitidas pelas maio e junho.

\begin{tabular}{c|c|c|c|c}
\hline & \multicolumn{2}{|c|}{ MAIO } & \multicolumn{2}{c}{ JUNHO } \\
\hline ESTABELECIMENTO & FUNGO & FLUORESCÊNCIAS & FUNGO & FLUORESCÊNCIA \\
\hline A & Negativo & - & A. niger & AZUL \\
\hline B & Mucor spp & - & A. niger & VERDE \\
\hline C & A. niger & VERDE & A. niger & VERDE \\
\hline D & Negativo & - & Negativo & - \\
\hline E & Negativo & - & Negativo & - \\
\hline F & Negativo & - & A. niger & AZUL \\
\hline G & A. flavus & AZUL & A. niger & VERDE \\
\hline H & Penicillium spp & AZUL & Negativo & - \\
\hline I & A. niger & AZUL & A. niger & AZUL \\
\hline J & A. niger & AZUL & A. niger & AZUL \\
\hline K & A. niger & VERDE & Negativo & - \\
\hline
\end{tabular}

Apesar de não ter sido utilizado um padrão de comparação na técnica de cromatografia em camada delgada, além de se utilizar a revelação por luz UV, utilizou-se como contra prova a aspersão ácido sulfúrico seguido de aquecimento, pois este reage especificamente com a aflatoxina gerando-se um derivado de cor amarela fluorescente. A identificação de espécies produtoras de aflatoxinas através de microcultivo corroborou para esta confirmação.

Apenas a cromatoplaca do estabelecimento $G$ apresentou coloração amarela, indicando assim a presença de aflatoxina. Todas as placas não apresentaram coloração amarelo confirmando a ausência de aflatoxina, podendo se pela fluorescência e também pelo microcultivo, inferir presença de outra toxina, sugestiva de Ocratoxina.
Tabela 3 - Relacão dos fungos produtores de micotoxinas identificados com as fluorescências observadas nas cromatoplacas nos ensaios de contra prova com ácido sulfúrico.

\begin{tabular}{|c|c|c|c|c|c|c|}
\hline & \multicolumn{3}{|c|}{ MAIO } & \multicolumn{3}{|c|}{ JUNHO } \\
\hline ESTABELECIMENTO & FUNGOS & $\begin{array}{c}\text { TOXINA } \\
\text { SuGESTIVA }\end{array}$ & $\begin{array}{c}\text { FLUOR.:" } \\
\text { C.P." }\end{array}$ & FUNGOS & $\begin{array}{c}\text { TOXINA } \\
\text { SUGESTIVA }\end{array}$ & $\begin{array}{l}\text { FLUOR.*" } \\
\text { C.P." }\end{array}$ \\
\hline A & Negativo & Negativo & - & A niger & Ocratoxina & AZUL \\
\hline B & Mucor spp & - & - & A. niger & Ocratoxina & VERDE \\
\hline $\mathrm{c}$ & A. niger & Ocratoxina & VERDE & A. niger & Ocratoxina & VERDE \\
\hline D & Negativo & $\begin{array}{l}\text { Negativo } \\
\end{array}$ & - & $\begin{array}{l}\text { Negativo } \\
\end{array}$ & Negativo & - \\
\hline E & Negativo & $\begin{array}{l}\text { Negativo } \\
\end{array}$ & - & $\begin{array}{l}\text { Negativo } \\
\end{array}$ & $\begin{array}{l}\text { Negativo } \\
\end{array}$ & - \\
\hline $\mathrm{F}$ & Negativo & Negativo & - & A. niger & Ocratoxina & AZUL \\
\hline G & A. flavus & $\begin{array}{l}\text { Aflatoxina } \\
\end{array}$ & $\begin{array}{c}\text { AZUL/ } \\
\text { AMARELO }\end{array}$ & A. niger & Ocratoxina & VERDE \\
\hline H & $\begin{array}{l}\text { Penicillium } \\
\text { spp }\end{array}$ & Ocratoxina & AZUL & Negativo & Negativo & - \\
\hline I & A. niger & Ocratoxina & AZUL & A. niger & Ocratoxina & AZUL \\
\hline$J$ & A. niger & \begin{tabular}{|l} 
Ocratoxina \\
\end{tabular} & AZUL & A. niger & Ocratoxina & AZUL \\
\hline $\mathrm{K}$ & A. niger & Ocratoxina & VERDE & Negativo & Negativo & - \\
\hline
\end{tabular}

*Fluor: Fluorescência; *C.P: Contra prova

Foram analisadas 6 amostras de uvas passas escuras embaladas e 5 à granel no mês de maio, sendo que destas, 2 amostras embaladas e 4 à granel apresentaram resultado positivo para fungos toxigênicos. Essa análise foi repetida no mês de junho com 7 amostras embaladas e 4 à granel, apresentando resultados positivos em 4 amostras embaladas e 3 à granel.

De todas as amostras analisadas (22), 50\% apresentaram colonização por Aspergillus niger, e 4\% para Aspergillus flavus, 5\% para Penicillium spp, 5\% para Mucor spp e oito amostras (36\%) não apresentaram crescimento fúngico em relação ao meses de maio e junho de 2014.

No mês de maio foi identificada uma amostra positiva para A. flavus (12\%) uma para Penicillium spp (12\%), quatro amostras positivas para A. niger (49\%), uma amostra positiva para Mucor spp (12\%) e quatro amostras negativas (15\%). Devido ao fato do Mucor spp ser um fungo que pode ser encontrado tanto no solo como no ar, a amostra positiva para este gênero foi devido à contaminação, não sendo relevante para a pesquisa, pois este não é um fungo produtor de toxina.

Mucor spp é um fungo filamentoso de baixa virulência, causador de micoses oportunistas que são infecções cosmopolitas que convivem pacificamente com o hospedeiro, mas quando ocorre algum distúrbio do sistema imunodefensivo, desenvolve seu poder patogênico (SIDRIM \& MOREIRA, 1999).

Foi realizada a mesma análise no mês de junho dos mesmos estabelecimentos comerciais e foram identificadas apenas sete amostras positivas para A. niger (64\%) e quatro amostras negativas (36\%).
ISUNIARA
Volume $18, n^{\circ} 1$, julho de 2015

Revista Uniara $\cdot 185$ 
Conforme IAMANAKA et al. (2007) três espécies de Aspergillus: A. flavus, A. parasiticus e A. nomius são capazes de produzir aflatoxinas (B1, B2, G1 e G2) encontradas normalmente em vários produtos alimentícios, incluindo frutas secas (uvas passas escuras, figos, damascos). Estas são capazes de crescer em condições de temperatura, umidade relativado ar e umidade do produto quando são favoráveis.

O Laboratório de Análise Micotoxicológico (LAMIC) no Rio Grande do Sul analisou 70 amostras de frutas secas (uvas passas, damascos, ameixas secas e peras secas) comercializadas no Brasil no ano de 2002. As frutas secas apresentaram $71 \%$ de contaminação por aflatoxina (MALLMANN et al., 2002).

Segundo MÁRCIA e LÁZZARI (1998) são considerados fungos de armazenamento o Aspergillus spp, Penicillium spp e Mucor spp normalmente encontrados no solo e também em armazéns, moinhos, equipamentos e lugares onde os alimentos são armazenados, manuseados e processados

Devido ao fato das frutas secas terem amplo prazo de validade e conseqüentemente possibilidade de consumo durante todo o ano, as condições inapropriadas de armazenamentoao longo prazo, e o alto teor de açúcar, favorecem o crescimento de fungos incluindo também os micotoxigênicos (FERRACIN, 2007)

Segundo o trabalho realizado por TANIWAKI et al. (2004) as amostras de uvas passas escuras apresentaram alta frequência de infecção por A. niger produtor de ocrato-xina.

MAGNOLLI et al. (2004) estudaram 50 amostras de uvas passas escuras argentinas, sendo a espécie A. niger mais encontrada. Quanto à presença de ocratoxina, revelou se que $74 \%$ das amostras estavam contaminadas, sendo que em $28 \%$ por Aspergillus da espécie Nigri.

ABARCCA et al. (2003) analisaram uvas passas espanholas detectando se a presença de A. niger e A. carbonarius em $98 \%$ e $58 \%$ das amostras analisadas, respectivamente. Todos estes relatos são indícios de que a contaminação por A. niger em uvas passas é frequente.

É de extrema importância a análise destes alimentos, pois nem sempre a presença de fungos implica que toxina lado, a ausência de sinais visíveis de bolor não significa que o alimento está livre dos fungos produtores de toxinas (IAMANAKA; OLIVEIRA; TANIWAKI, 2010).

\section{CONCLUSÃo}

Fica evidente que a contaminação de uvas passas por fungos produtores de micotoxinas não é incomum, e que o Aspergillus da espécie Nigri são os principais responsáveis pela contaminação das frutas secas, sendo este produtor de ocratoxinas, hepatotóxicos no organismo humano.

O presente trabalho identificou a contaminação por este fungo tanto em uvas passas escuras á granel quanto nas embaladas no próprio estabelecimento comercial na qual não foi observada alterações significativas de amostras positivas no mês de maio em relação à análise comparativa realizada em junho.

A análise microbiológica foi confirmatória para a identificação dos fungos produtores de micotoxinas, porém o método de cromatografia em camada delgada apenas possibilitou a detecção da ausência ou presença de alguma toxina que apresentaram fluorescência azul ou verde, pelo fato de não terem sido utilizados padrões, a identificação específica das micotoxinas foi impossibilitada. A contra prova realizada permitiu apenas distinguir a aflatoxina de ocratoxina.

\section{REFERÊNCIAS}

ABARCA, M. L.; ACCENSI, F.; BRAGULAT, M. R.; CASTELLA, G.; CABANES, F. J. Aspergillus carbonarius as the main source of ochratoxin A contamination in dried vine fruits from the Spanish market. Journal of Food Protection, v. 66, n. 3, p.504-506, Mar. 2003.

BATATINHA, M. J. M.; SANTOS, M. M.; BOTURA, M. B.; ALMEIDA, G. N.; DOMINGUES, L. F.; KOWALSKI, C. H.; MALLMANN, C. A. Ocorrência de Afla toxinas em amendoim e seus produtos comercializados no estado da Bahia durante o ano de 2002. Revista Instituto Adolfo Lutz, v. 62, n. 3, p. 183-187, 2003.

BRUEGEL, P. Mycotoxins: Risks in Plant, Animal, and Human Systems. Council for Agricultural Science and Technology, n. 139, p. 1-199, Jan. 2003.

CALDAS, D. E.; SILVA, C. S.; OLIVEIRA. N. J. Aflatoxinas e Ocratoxina A em alimentos e riscos para a saúde humana. Revista Saúde Pública. v. 36, n.3, p.319-323, Mar. 2002

FAVILLA, M.; PASCALE, M.; RICELLI, A.; EVIDENTE, A.; AMALFITANO, C.; ALTOMARE, C. Inhibition of Species of the Aspergillus Section Nigri and Ochratoxin A Production in Grapes by Fusapyrone. Applied And Environmental Microbiology, v.74, n.7, p. 2248 2253. Apr. 2008

FERRACIN, L. M. Identificação e caracterização molecular de isolados do agregado Aspergillus niger obtidos de frutas secas. 2007. 79 f. (DissertaçãoMestrado em Genética e Biologia Molecular)Mestrado em Genética e Biologia Molecular)2007.

FRANCO, B. D. G. M. LANDGRAF, M. Microbiologia dos alimentos. São Paulo: Atheneu, 2000.

FREIRE, S. C. O.; VIEIRA, I. G. P.; GUEDES, M. I. F.; MENDES, F. N. P. Micotoxinas:Importância na Alimentação e na Saúde Humana e Animal. Embrapa Agroindústria
Tropical, n.1, p.9-38, Out. 2007.

IAMANAKA, B. T.; OLIVEIRA, I. S.; TANIWAKI, M. H. Micotoxinas em alimentos. In: Anais da Academia Pernambucana de Ciência Agronômica, 2010, Recife.REUNIÃO DA ACADEMIA PERNAMBUCANA DE CIÊNCIA AGRONÔMICA, 2. Anais.. Recife. p. 138-161.

IAMANAKA, B. T.; TANIWAKI, M. H.; MENEZES, H. C.; VICENTE, E.; LEITE, R. S F. TANIWAKI, M. H. Aflatoxigenic fungi and aflatoxins occorrence in sultanas and dried figs commercialized in Brazil. Food Control, v. 18, p. 454-457, Dec. 2007

KOVACS, M. Nutritional health aspects of mycotoxins. Orvosi Hetilap, p. 1739 1746, Ago. 2004. Disponível em: <http://europepmc.org/ abstract/med/15493122> Acesso: 18 Jul. 2014.

KWIATKOWSKI, A.; ALVES, A. P. F. Importância da detecção e do controle de aflatoxinas em alimentos. SaBios Revista Saúde e Biologia. v. 2, n. 2, p. 45 54, 2007.

MAGNOLI, C.; ASTORECA, A.; PONSONE, L.; COMBINA, M.; PALACIO, G.; ROSA,C. A. R.; DALCERO, A. M. Survey of mycoflora and ochratoxin A in dried vine fruits from Argentina markets. Letters in Applied Microbiology. v. 39, p. 336 331Jun. 2004.

MALMANN, C.A.; KOWALSKI, C.H.; ALMEIDA, C. A. A.; DILKIN, P.; MURMANN, L.; DILKIN, M. Aflatoxinas em nozes e frutas secas comercializadas no Brasil. 2002. Disponível em: <http://www.lamic.ufsm.br/papers/112z.pdf $>$ Acesso: 22 Ago. 2014.

MÁRCIA, B. A.; LÁZZARI, F.A. Monitoramento de fungos em milho em grão, grits e fubá. Ciência e Técnologia de Alimentos, v. 18, n. 4, Out/Dez. 1998. 
MASLINKIEWICZ, A.; FREITAS, D. R. J. Micotoxinas: o que são e quais suas conse quências para o homem?. SB RURAL, Chapecó, 17 Mai. 2012. Caderno Rural, p. 1 4. Disponível em: <http://www.ceo.udesc.br/arquivos/id submenu/285/caderno udesc 087.pdf $>$. Acesso em: 22 Set. de 2014

MENEGHELLO, E. R.; JORGE, J. R.M.; OLIVEIRA, A.V. Identificação e Pesquisa de espécies de fungos Aspergillus nige e Aspergillus carbonarius encontradas em frutas secas vendidas no comércio de Maringá-PR. In: VI MOSTRA INTERNA DE TRABALHOS DE INICIAÇÃO CIENTÍFICA, 2012, Maringá-PR. Identificação e Pesquisa de espécies de fungo.,6. Anais Eletrônicos.. aspergillus. Níger e Aspergillus Carbonarius encontrados em frutassecas vendidas no comércio de Maringá PR. Maringá PR6, Maringá: CESUMAR, 2012 p.1 9. Disponível em: $<$ http://www.cesumar.br/prppge/pesquisa/ mostras/vi mostra/jessica raynne moura jorge 2.pdf $>$ Acesso em: 5 Set. 2014.

AS MICOTOXINAS. Revista Food Ingredients Brasil. N. 7, p32- 40, 2009. Disponível em: $<$ http:// www.revista-fi.com/materias/90.pdf $>$ Acesso em 24 Jun. 2014.

MOREAU, R. L. M.; SIQUEIRA, M. E. P. B. Toxicologia Análitica: Ciências Farmacêuticas.1 ed. Rio de Janeiro: Guanabara Koogan, 2001.p.199.

OLIVEIRA, J. N.; OLIVEIRA, A. V.; MENEGHELLO, E. R. Análise Molecular de espécies de Aspergillus contaminantes de uvas vendidas no comércio de Maringá PR. Iniciação Científica CESUMAR. v. 15, n. 2, p. 157-163, Jul/ Dez. 2013.

PEREYRA, C. M.; CAVAGLIERI, L. R.; CHIACCHIERA, S. M.; DALCERO, A M. Fungi and Mycotoxins in Feed Intended for Sows at Different Reproductive Stages in Argentina. Veterinary Medicine International, v. 2010, p.1-7, Apr. 2010.

PERRONE, G.; SUSCA, A.; COZZI, G.; EHRLICH, K.; VARGA, J.; FRISVAD, J. J. C.;MEIJER, M.; NOONIM, P.; MAHAKARNCHANAKUL,W.; SAMSON, R. A. Biodiversity of Aspergillus species in some important agricultural products. Studies in Mycology, v.59, n.09, p.53-66, 2007.

SANTOS, C. C. M.; LOPES, M. R. V; KOSSEKI, S. Y. Ocorrência de aflatoxinas em amendoim e produtos de amendoim comercializados na região de São José do Rio Preto/SP. Revista Instituto Adolfo Lutz, v.60, n.2, p. 153-157, 2001

SIDRIM, J. J. C.; MOREIRA, J. L. B Fundamentos clínicos e laboratoriais da micologia médica. 1 ed. Rio de Janeiro: Guanabara Koogan, 1991. p. 1-274.

TANIWAKI, M. H.; MARTINS, C. P.; IAMANAKA, B. T.; LEITE, R. S. S. F.; VICENTE, E.; OKAZAKI, M.M. Aspergillus niger e Aspergillus carbonarius produtores de ocratoxina a em alimentos: ocorrência, significado e fatores que afetam a sua presença. REVNET DTA Revista Eletrônica de Epidemiologia das Doenças Transmitidas por Alimentos. v. $4, \mathrm{n} .1$ 2004. Disponível em: <ftp://ftp.cve.saude.sp.gov. $\mathrm{br} /$ doc_tec/hidrica/revp04_vol4n1.pdf $>$ Acesso em: 19 Set. 2014

WELKE, J. E.; HOELTZ, M.; NOLL, I. B. Aspectos relacionados à presença de fungos toxigênicos em uvas e ocratoxina A em vinhos. Ciência Rural, v.39, n.8, p.2567-2575, Nov. 2009.

ZAITZ, C.; RUIZ, L. R. B.; SOUZA, V. M. S. Atlas de Micologia Médica: Diagnóstico Laboratorial. 2 ed. Rio de Janeiro: MEDSI, 2004. p.2-169.

\section{PESQUISA DE BIOINDICADORES EM \\ SALSICHAS VENDIDAS A GRANEL EM SUPERMERCADOS DO NOROESTE PAULISTA}

Bruna Tavares Bruno ${ }^{1}$

Livia Alegria dos Santos

Cátia Rezende

\section{RESUMO}

A pesquisa de Escherichia coli e Salmonella spp. nos alimentos é um importante bioindicador higiênicosanitário, indicando contaminação fecal. A salsicha é um alimento muito consumido e de fácil preparo, entretanto, se manipulada de maneira inadequada, pode se tornar uma excelente fonte de contaminação e proliferação de micro-organismos. Para se analisar a presença dessas bactérias, foram coletadas 32 amostras de salsichas em supermercados de pequeno e grande porte. A análise microbiológica quantitativa para Escherichia coli foi realizada em ágar EMB e para Salmonella spp., em ágar SS. Colônias suspeitas foram identificadas por testes bioquímicos. Do total de amostras coletadas, 50\% apresentaram crescimento de Escherichia coli e para Salmonella spp não houve crescimento. Esses dados comprovam a necessidade de uma maior fiscalização higiênico-sanitária nos estabelecimentos comerciais e uma maior atenção da população aos alimentos que estão sendo ingeridos.

Palavras-chave: Bioindicador; Contaminação; Doenças Intestinais. 